\title{
Effect of Mud Bath on Autonomic and Respiratory Variables in Normal Healthy Volunteers- A Randomized Control Trial
}

\author{
Dr. N V Gnanadeep T ${ }^{1}$, Dr. Sujatha Dinesh ${ }^{2}$, Dr. Prashanth Shetty ${ }^{3}$ \\ ${ }^{I}$ (Post Graduate, Division Of Natural Therapeutics, Department Of Post Graduate Studies, SDM College Of \\ Naturopathy And Yogic Sciences, India) \\ ${ }_{2}^{2}$ (Dean, Division Of Natural Therapeutics, Department Of Post Graduate Studies, SDM College Of Naturopathy \\ And Yogic Sciences, India) \\ ${ }^{3}$ (Principal, SDM College Of Naturopathy And Yogic Sciences, India)
}

\begin{abstract}
:
Background and Objectives: Mud therapy is a system of Naturopathic medicine where treatment is given using various forms of mud. Full mud bath is one of the treatment used in Mud therapy for the management of various disorders. Hence present study aims to evaluate the physiological effects of full mud bath. Materials and Methodology: 60 male healthy volunteers were recruited for the study. Subjects were randomly allocated into two groups, full mud bath and cold wet wrap. Results: In the present study, among two groups full mud bath group shown increase in The square root of the mean squared difference between adjacent $N-N$ intervals (RMSSD), Consecutive normal sinus (NN) intervals exceeds 50 ms (NN50), High Frequency (HF), Mean of R-R interval (Mean RR). Heart Rate (HR), Very low frequency (VLF), Low frequency (LF), Systolic Blood Pressure $(S B P)$, Diastolic Blood Pressure (DBP), and Temperature (TEMP) reduced slightly after the treatment. Conclusions: Application of full mud bath on the body enhances the sympathetic activity and could have a role in maintaining the cardiac tone and preventing various cardiovascular ailments, where as a cold wet wrap enhances parasympathetic activity.
\end{abstract}

Keywords: Autonomic Nervous System, Cold wrap, Heart rate variability, Mud bath, Naturopathy.

\section{Introduction}

Naturopathic medicine is the art and science of disease diagnosis, treatment and prevention using natural therapies including: Botanical medicine, Clinical nutrition, Hydrotherapy, Homeopathy, Naturopathic manipulation, Traditional Chinese medicine/Acupuncture, Prevention and lifestyle counselling. ${ }^{[1]}$

One of the unique properties of mud is that, it can absorb the heat and toxins from the body and eliminate these toxins in different ways. Mud therapy is a very simple and cost effective treatment procedure in Naturopathy. The mud used for treatment purpose should be collected from $122 \mathrm{~cm}$ to $153 \mathrm{~cm}$ depth from the surface of the ground and should be very clean, pure and free from all the contaminations. ${ }^{[2]}$

Mud starts its action on the body right from the moment it is applied and patients observe the changes in the symptoms immediately. Mud is used in various ways to treat different diseases, in the form of packs, compresses and full body applications. Full mud bath is one of the most popular and important treatment modalities in the Naturopathy hospitals. In this type of mud applications, mud is applied all over the body, followed by cleansing by a cold shower. The duration of the treatment is 40 minutes followed by wash with cold water for 10 minutes. The chemical analysis of the mud has revealed that mud contains hydrophilic organic compounds like humic acid, fulmic acid and ulmic acid. It also contains organic substances like fatty acids. Because of these properties and components of the mud it has many therapeutic characters like improving membrane electrical conductance, absorption, hyperthermia, activating hydropoietic glands and hormones in the body. However the composition and compounds of the mud and its properties change according to the area of availability.

Mud therapy either as mud bath or mud pack is used to treat the conditions like fever, diarrhoea, piles, dysentery, constipation, anxiety, conjunctivitis, headache, allergy, errors of refraction in the eyes. It is useful to treat the cardiovascular, musculoskeletal, gynaecological, dermatological and autoimmune diseases etc. Worldwide mud bath is taken for relaxation and luxurious purposes. ${ }^{[2,3]}$

Though mud bath has been used extensively for various conditions, the actual mechanism of how a mud bath works remains unclear. This study is conducted to explain the physiological effects of mud bath in healthy volunteers. 


\subsection{Subjects}

\section{Methodology}

Sixty male normal healthy volunteers of ages ranging between 18 to 28 years were recruited.

\subsubsection{Study Group}

Male Students from Sri Dharmasthala Manjunatheshwara (SDM) College of Naturopathy and Yogic Sciences, Ujire, Dakshina Kannada were recruited for the study.

\subsubsection{Inclusion criteria}

- Age: 18 to 28 years.

- Subject who is willing to participate in the study.

- Gender: Male.

The female subjects are excluded because this study requires to assess the immediate effects after intervention.

\subsubsection{Exclusion criteria.}

- The following criteria were used to exclude the volunteers.

- Any health disorders (in order to determine the health status of the subjects, in all cases a routine case history was taken and a routine clinical examination was conducted).

- Those who were taking medication which could influence autonomic function e.g., phenylpropanolamine as a common cold remedy. ${ }^{[4]}$

- Those who are consuming alcohol and nicotine except caffeine.

\subsubsection{Consent form}

The subjects were instructed about the study and all subjects who are willing to take part in the study were considered. A signed informed consent was obtained from each individual. Institutional Ethical Committee approved the study.

\subsection{Study setting}

\subsubsection{Setting for assessments and Interventions}

Subjects were randomly allocated into two groups using computerized generated random number table. For (group 1) full mud bath was given for 45 minutes followed by wash with cold water, for (group 2) cold wet wrap was given for 45 minutes followed by wash with cold water for 15 minutes. Assessments were done 5 minutes before and 5 minutes immediately after the intervention.

\subsection{Design}

The study adopted was Randomized Control Trial. 60 healthy volunteers were divided in to two groups. Group 1 received full mud bath, group 2 received cold wet wrap. All the subjects were assessed before and after the treatment.

\subsection{Assessments}

Condition of recordings: The subjects were seated on a chair recording leads were connected to the four-channel polygraph equipment (BIOPAC, Montana, USA; model No: BSL 4.0 MP 36) and monitored on a closed circuit TV. Instructions were given to the subjects to remain relatively undisturbed during the session.

\subsubsection{Variables Studied}

The word variable has been used to denote 'measurement or attribute on which observations are made ${ }^{[5]}$ Hence in the present thesis the assessments measures have been described as variables.

\section{Autonomic and respiratory variables.}

\subsubsection{Rationale for studying autonomic and respiratory variables}

In the present study, the autonomic variables measured were heart rate, and heart rate variability (HRV), Respirogram, blood pressure, pulse rate and body temperature. The HRV spectrum is believed to be an useful indicator of cardiac sympathetic activity (reflected by low frequency [LF] band power values) and parasympathetic activity (reflected by high frequency $[\mathrm{HF}]$ band power values). ${ }^{[6]}$

\subsubsection{Heart rate and Heart rate variability spectrum (HRV)}

Heart rate variability (HRV) describes the variation between consecutive heartbeats. The regulation mechanisms of HRV originate from the sympathetic and parasympathetic nervous systems in addition to other 
controls and hence, HRV is used as a quantitative marker of the autonomic control over the heart. The electrocardiogram [EKG] was recorded using standard bipolar limb lead II configuration and an AC amplifier with $1.5 \mathrm{~Hz}$ high pass filter and $75 \mathrm{~Hz}$ low pass filter settings (BIOPAC, Montana, USA; Model No: BSL 4.0 MP 36). The ECG was digitized using a 12-bit analogue-to-digital converter (ADC) at a sampling rate of 500 $\mathrm{Hz}$. The R waves were detected to obtain a point event series of successive R-R intervals, from which the beatto-beat heart rate series was computed. The data recorded was visually inspected off-line and only noise free data was included for analysis. The heart rate was obtained based on R-R inter beat interval analysis. Schematic representation of ECG and Heart rate variability (HRV) are presented in. According to guidelines of the Task force of the European Society of Cardiology and the North American Society of Pacing and Electrophysiology, the low frequency and high frequency values were expressed as normalized units. ${ }^{[7]}$

\subsubsection{Respirogram}

Respiration was recorded using a volumetric pressure transducer [stethograph] connected to AC amplifier of the polygraph and with sensitivity set as required. The stethograph was fixed around the trunk about $8 \mathrm{~cm}$ below the lower costal margin as the subject sat erect. ${ }^{[8]}$

\subsubsection{Digital Pulse Volume}

For this recording, the transducer was placed on the volar surface of the digital phalanx of the left thumb to record the digital pulse volume (DVP), also called the finger plethysmogram. Photoplethysmography allowed normal non-invasive recording of arterial blood volume pulse. The Photoplethysmogram described the changes in the absorption of light by haemoglobin using waveforms. The amplitude of the DVP was sampled from the peak of the pulse waves at 30 -second intervals and presented in $\mathrm{mm} .{ }^{[8]}$

\subsubsection{Blood Pressure}

The blood pressure was recorded with a sphygmomanometer by auscultation over the right brachial artery. ${ }^{[9]}$

\subsubsection{Body temperature}

The Mercury-in-glass thermometers have been used as a standard tool to measure the temperature since 1867. ${ }^{[10]}$ The body temperature will be recorded with a thermometer by placing under left axillary arm pit.

\subsection{Intervention}

\subsubsection{Full mud bath for group 1}

- Black coloured mud which will be collected from rivers banks

- That mud will be collect from $10 \mathrm{~cm}$ below the ground level.

- That mud should be free from impurities, composts or pebbles.

- The mud will be finely sieved.

- It will be kept under sunlight for at least one day.

- Sufficient quantity of cold water should be added to the mud so as to making it a paste like substance.

- Person with minimum dressing.

- The paste like mud will be applied all over the body in standing position. The mud should not slide off from the surface of the body.

- Mud should apply evenly and cover all over the body for 10 minutes.

- No movements should be undertaken once application is done.

- After the application of mud leave the person for a period of 40 minutes.

- After 40 minutes mud should be wash with cold water for 10 minutes ..$^{11-16]}$ Temperature: 12.7 to $18.3^{0} \mathrm{C}^{[16]}$

\subsubsection{Cold wet wrap for group 2}

- Rinse the cloth in the cold water.

- Lightly squeeze the cloth. Wrap around the body in standing position for 10 minutes.

- No movements should be undertaken once application done.

- After the application of cold wet wrap, leave the person for a period of 40 minutes.

- After 45 minutes wash with cold water for 10 minutes. ${ }^{[12-15]}$ Temperature: 12.7 to $18.3^{0} \mathrm{C}^{[16]}$

\subsubsection{Materials Used}

- Channel Polygraph (BIOPAC, Montana, USA; Model No: BSL 4.0 MP 36).

- Sphygmomanometer. 
- Thermometer.

- White cotton cloth.

- Cleaned wet mud.

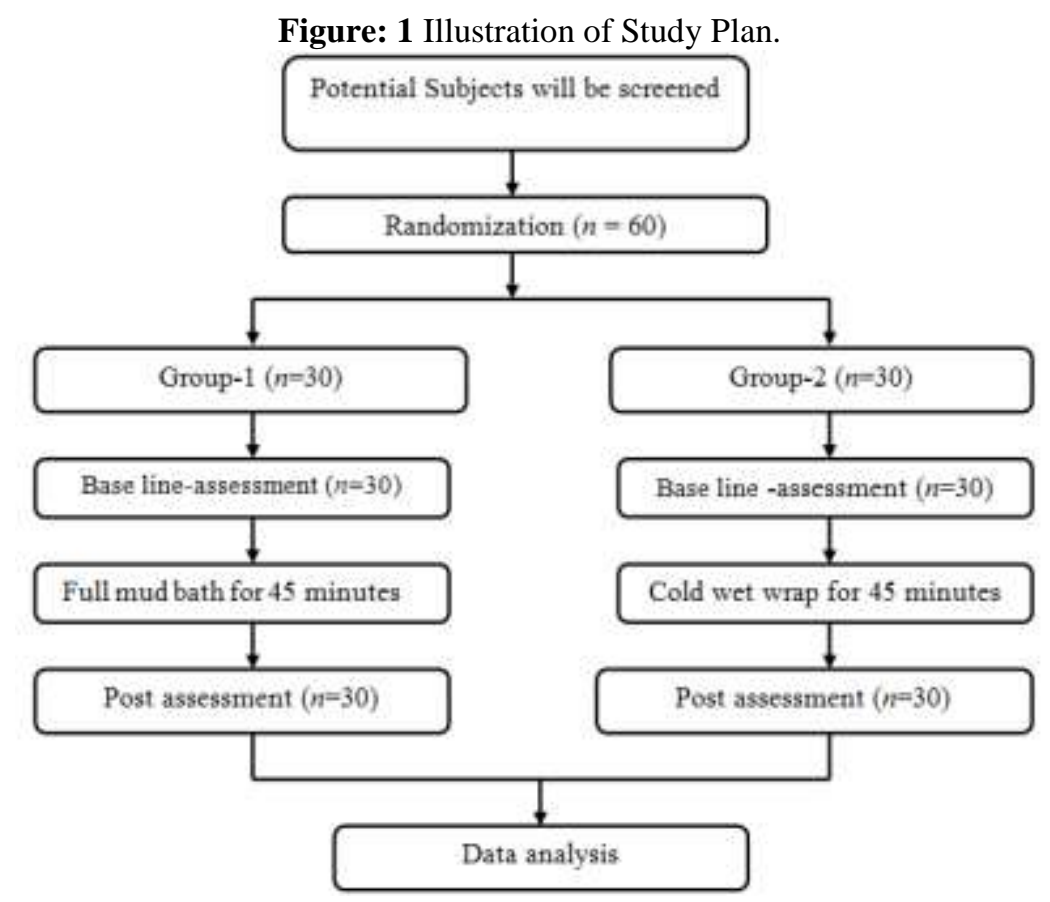

Figure 2: Full mud bath

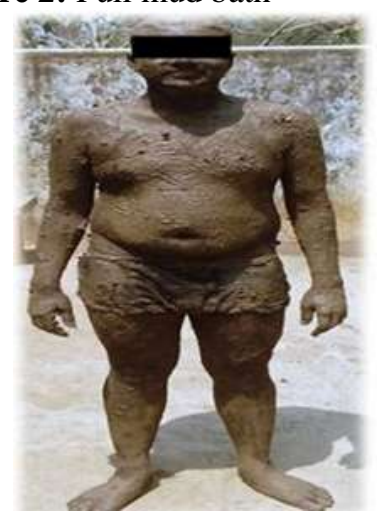

Figure 3: Cold wet wrap

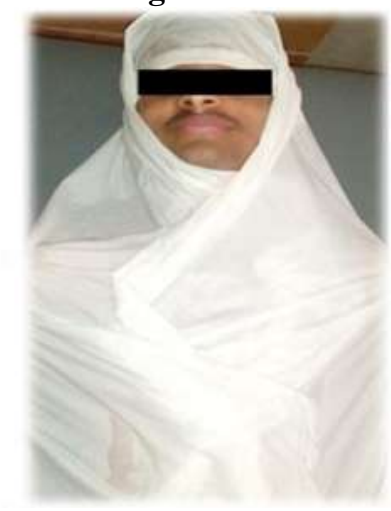

\subsection{Data Extraction.}

2.6.1. Autonomic and respiratory variables.

\subsubsection{Heart Rate and Heart Rate Variability (HRV).}

The heart rate in beats per minute was calculated by counting the R waves of the QRS complex in the EKG in successive epochs of 60 seconds and averaged for each 5- minute block period. The HRV power spectrum was obtained using fast Fourier transform analysis (FFT). The energy in the HRV series of the following specific bands were studied, viz., the very low frequency component $(0.0-0.05 \mathrm{~Hz})$, low frequency component $(0.05-0.15 \mathrm{~Hz})$, and high frequency component $(0.15-0.50 \mathrm{~Hz})$.

The low frequency and high frequency values were expressed as normalized units, which represent the relative of each power component in proportion to the total power minus VLF component [LF norm $=\mathrm{LF} /$ $($ total power-VLF $) \times 100 ; \mathrm{HF}$ norm $+\mathrm{HF} /($ total power $-\mathrm{VLF}) \times 100)$ ] for a typical trace of HRV spectrum.

\subsubsection{Respiratory Rate.}

Readings were obtained for every 60 seconds as number of cycles per minute. The readings were averaged for each five-minute block period. 

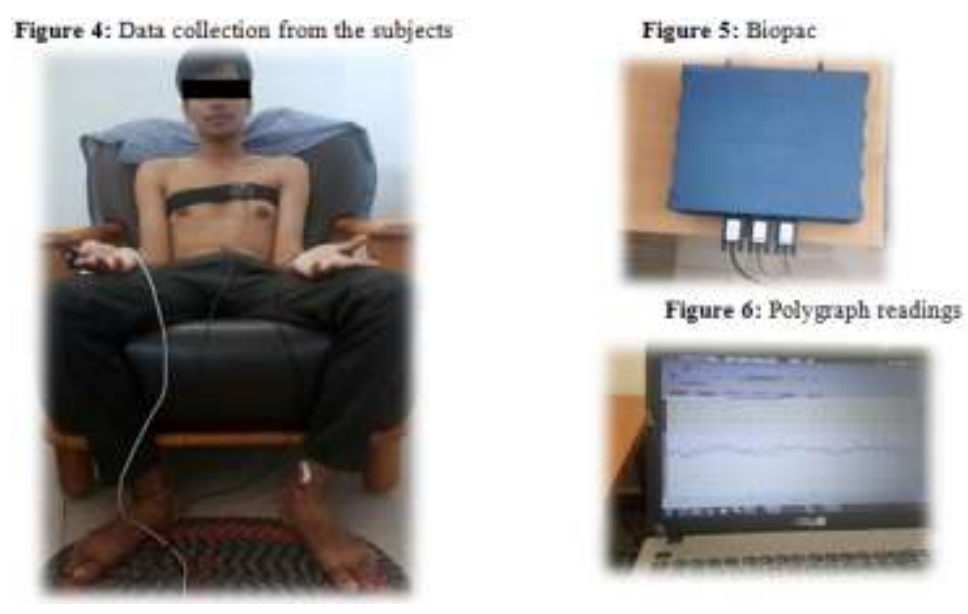

\subsection{Stastical analysis.}

The raw data obtained from each subject in each recording session were tabulated separately. The distribution of the data and normality assumption were estimated, than the group's median, mean, standard deviation and Sw p- values were calculated for all the variables. Kruskal -Wallis One-Way Analysis of Variance (Mann-Whitney U test value) for inter group comparison between the full mud bath group and wet wrap group. Statistical analysis was done using Statistical Package for Social Sciences (SPSS 21.0).

\section{Results}

In the present study comparison among two groups full mud bath group shows an increase in RMSSD, NN50, HF, Mean RR after the treatment. Whereas HR, VLF, LF, SBP, DBP temperature reduced slightly, PR, RR remained unchanged. Suggests arousal of sympathetic activity following full mud bath.

Cold wet wrap group shows an increase in Mean RR, VLF, and LF after the treatment. Whereas HR, RMSSD, NN50, HF, DBP reduced slightly, PR, RR, SBP, temperature remained unchanged .Suggests arousal of parasympathetic activity following cold wet wrap.

Comparing the two groups, reduction in HR, LF, VLF and increase in RMSSD, NN50, Mean RR, HF were observed in the full mud bath group than the cold wet wrap. Reduction in heart rate, RMSSD, NN50 and following the cold wet warp suggest parasympathetic dominance. There is an increase in the mean RR, RMSSD, NN50, HF and reduction in HR, VLF, and LF which indicates sympathetic dominance in the full mud bath group.

Although the results of individually groups are show some changes, but there is no significance in the form of $(\mathrm{p}<0.05)$ when compared between the inter group that is full mud bath and cold wet wrap.

Table 1: Kruskal -Wallis One-Way Analysis of Variance (Mann-Whitney U test value) for post inter group comparisons.

\begin{tabular}{|c|c|c|c|c|}
\hline $\begin{array}{c}\text { VARIABLES } \\
\text { (Post Variables) }\end{array}$ & $\begin{array}{c}\text { GROUP I (Mud } \\
\text { bath) Sample } \\
\text { median (SD) }\end{array}$ & $\begin{array}{c}\text { GROUP 2 (Cold wet } \\
\text { wrap) Sample } \\
\text { median (SD) }\end{array}$ & $\begin{array}{c}\text { Mann- } \\
\text { Whitney U test } \\
\text { values }\end{array}$ & p-VALUE \\
\hline HR & $67.00(9.14)$ & $68.62(6.92)$ & 404.50 & 0.50 \\
\hline RR & $15.48(1.52)$ & $15.90(1.18)$ & 465.50 & 0.82 \\
\hline PR & $86.47(3.37)$ & $85.85(1.58)$ & 508.50 & 0.39 \\
\hline MEAN RR & $897.20(186.75)$ & $881.10(153.70)$ & 489.00 & 0.56 \\
\hline RMSSD & $58.95(32.58)$ & $42.85(96.24)$ & 516.00 & 0.33 \\
\hline NN50 & $117.00(69.86)$ & $67.50(67.39)$ & 575.50 & 0.06 \\
\hline pNN50 & $29.95(17.74)$ & $20.75(35.68)$ & 497.50 & 0.48 \\
\hline VLF & $32.60(19.00)$ & $35.65(16.13)$ & 414.00 & 0.59 \\
\hline LF & $55.00(18.75)$ & $60.50(19.02)$ & 377.50 & 0.28 \\
\hline HF & $47.15(19.80)$ & $40.15(65.03)$ & 526.50 & 0.26 \\
\hline LF/HF & $1.23(1.11)$ & $1.54(2.55)$ & 374.50 & 0.26 \\
\hline SBP & $115.50(9.61)$ & $110.00(11.98)$ & 448.00 & 0.98 \\
\hline DBP & $73.00(8.99)$ & $73.00(14.16)$ & 507.50 & 0.39 \\
\hline TEMP & $98.10(0.70)$ & $98.30(0.52)$ & 335.00 & 0.09 \\
\hline
\end{tabular}

$<0.05$ Considered has a significant.

$\mathrm{SD}=$ Standard deviation, $\mathrm{HR}=$ Heart rate, $\mathrm{RR}=$ Respiratory rate, $\mathrm{PR}=\mathrm{Pulse}$ rate, MEANRR= Mean of $\mathrm{R}$ $\mathrm{R}$ interval, RMSSD=The square root of the mean squared difference between adjacent $\mathrm{N}-\mathrm{N}$ intervals, NN50=Consecutive normal sinus (NN) intervals exceeds $50 \mathrm{~ms}$, pNN50=The fraction of consecutive NN 
intervals that differ by more than $50 \mathrm{~ms}$, VLF=Very low frequency power, LF=Low frequency Power, HF=High frequency power, $\mathrm{LF} / \mathrm{HF}=\mathrm{Low}$ frequency/High frequency ratio, $\mathrm{SBP}=$ Systolic blood pressure, DBP=Diastolic blood pressure and TEMP=Temperature.

\section{Discussion}

The main aim of the study was to evaluate and compare the physiological effect of full mud bath and cold wet wrap and evaluate their respective changes on various autonomic functions viz. Heart rate, Heart Rate Variability, Respiratory rate, Pulse rate, Body temperature and Blood pressure in normal healthy volunteers. All the 60 subjects underwent the intervention for the duration of 45 minutes in the sitting posture .There were no adverse effects reported during or after the intervention.

Results of the individuals who underwent full mud bath group showed increase in RMSSD, NN50, HF, and Mean RR after the treatment. Whereas HR, VLF, LF, SBP, DBP and body temperature reduced slightly, RR remained unchanged. These findings suggest an arousal of sympathetic activity following full mud bath.

Individuals who underwent cold wet wrap showed an increase in mean RR, VLF, and LF after the treatment. Whereas HR, RMSSD, NN50, HF, DBP reduced slightly, RR, SBP and body temperature remained unchanged. These findings suggests an arousal of parasympathetic activity following cold wet wrap.

Comparing the two groups, a reduction in HR, LF, VLF and increase in RMSSD, NN50, Mean RR, and HF was observed in the full mud bath group in contrast to the cold wet wrap. Reduction in Heart Rate, RMSSD, NN50 and increase in mean RR, VLF, and LF following the cold wet wrap suggest parasympathetic dominance. An increase in the mean RR, RMSSD, NN50, HF and reduction in HR, VLF, and LF following the full mud bath indicates sympathetic dominance.

No changes in the temperature and respiratory rate were seen between and within both the groups, these results are similar with a study done by Dominique Poensin et al. ${ }^{[17]}$

The probable mechanism of action indicating a sympathetic dominance might be due to peripheral vasodilatation following an exposure to cold temperature. Effects of full mud bath could also be thermic and chemical in origin. A study suggests that there is a significant mineral and percutaneous cationic exchange along with other chemical changes taking place upon the application of mud on the human body. ${ }^{[18]}$

Although individual results of full mud bath indicates a sympathetic dominance, and that of a cold wet wrap indicating parasympathetic dominance, there is no strong evidence in the form of significant $(p<0.05)$ when either groups are compared which could be used for clinical relevance.

\section{Conclusion}

Application of full mud bath on the body enhances the sympathetic activity and has a role in maintaining the cardiac tone and preventing various cardiovascular ailments. Whereas a cold wet wrap enhances parasympathetic activity and can be applied effectively to treat stress, insomnia and anxiety. Though there was no significant changes observed in the form of $p$ value but there was a considerable change in the autonomic nervous system.

\subsection{Limitations of the Study.}

The main limitation of the study is that, there was lack of control over the variation in atmospheric temperature.

\section{Acknowledgements}

We thank the participants who participated for their willingness.

\section{References}

[1]. Hough H, Dower C, O’Neil E, Profile of a profession. san francisco: naturopathic practice (San Francisco, CA: Center for the Health Professions, University of California, 2001).

[2]. Rajiv R, Therapeutic uses of mud therapy in naturopathy, Indian journal of traditional knowledge, 11, 2012, 556-559.

[3]. Chadzopulu A, Adraniotis J, Theodosopoulou E, The therapeutic effects of mud, Progress in Health Sciences, 1, 2011, $132-136$.

[4]. Lake C R, Chernow B, Zaloga G, Labow J, Quirk R, Hedges S M, The effects of phenylpropanolamine on human sympathetic nervous system function, J Neuro Psy Pharma, 1(2), 1988, 163-168.

[5]. Altman D G, Gore S M, Gardner M J, Pocock S J, Statistical guidelines for contributors to medical journals, BMJ, 286, 1983, 14891493.

[6]. Task Force of the European Society of Cardiology and the North American Society of Pacing and Electrophysiology, Heart rate variability: Standards of measurement, physiological interpretation and clinical use, J. Circulation, 93, 1996, 1043-1065.

[7]. Zhao S, Xie L, Hu H, Xia J, Zhang W, Ye N, et al., A study of neonatal swimming (water therapy) applied in clinical obstetrics, J Matern Fetal Neonatal Med,17(1), 2005, 59-62.

[8]. Telles, S, Nagarathna R, Nagendra H R, Physiological measures during right nostril breathing, J Alt Com Med, 2(4), 1996, 479484.

[9]. Manjunath, N K, Telles S, Effect of Sirsasan (head stand) practice on autonomic and respiratory variables, Ind J Phys and Pharm, 47(1) 2003, 34-42.

[10]. Fulbrook P, Core temperature measurement in adults: a literature review, J Adv Nurs, 18, 1993, $1451-1460$.

DOI: $10.9790 / 0853-1509055864 \quad$ www.iosrjournals.org $63 \mid$ Page


[11]. Shakuntala S Raheja, Srhibani S Raheja, All about healthy living with naturopathy (Delhi, Vikas publishing house PVT LTD, 1998).

[12]. Ravindra V, Hema J, Prakash D, Nature cure for all (Pune: Nisargopchar ashram, Urulikanchan, Pune. Pace communication, 2007).

[13]. Binodini D, Nature cure and health care, (Delhi:, B. Jain publishers (p) ltd, 2007).

[14]. Institute of Naturopathy and Yogic Sciences, Nature Cure Treatment, (Bangalore, Institute of Naturopathy and Yogic Sciences, Brindavan printers and publishers private limited, 1994).

[15]. Chandrasekhar T, Nature Cure for Obesity, (Pune, Nisargopchar gramsudhar trust, nature cure, Urulikanchan, 2010).

[16]. Kellogg J.H, Rational hydrotherapy, (Pune, National Institute Of Naturopathy, Dept. Of AYUSH, Ministry of Health and FW. Govt. of India, Bapubhavan, Tadiwala Road, 2005).

[17]. Dominique P, Patrick H, Carpentier, Christiane F, Sylvie G, Effect of mud pack treatment on skin micro circulation, Joint Bone Spine, 70, 2003, 367-370.

[18]. Fernando V, Antonio B, Pier G J, Massimo S, Thermal muds: Perspectives of innovations, Applied Clay Sciences, 36, 2007, 141-7. 